\title{
Local Spectral Density and Vacuum Energy Near a Quantum Graph Vertex
}

\author{
Stephen A. Fulling
}

\begin{abstract}
The delta interaction at a vertex generalizes the Robin (generalized Neumann) boundary condition on an interval. Study of a single vertex with $N$ infinite leads suffices to determine the localized effects of such a vertex on densities of states, etc. For all the standard initial-value problems, such as that for the wave equation, the pertinent integral kernel (Green function) on the graph can be easily constructed from the corresponding elementary Green function on the real line. From the results one obtains the spectral-projection kernel, local spectral density, and local energy density. The energy density, which refers to an interpretation of the graph as the domain of a quantized scalar field, is a coefficient in the asymptotic expansion of the Green function for an elliptic problem involving the graph Hamiltonian; that expansion contains spectral/geometrical information beyond that in the much-studied heat-kernel expansion.
\end{abstract}

\section{Introduction}

A topic of perennial and renewed interest in quantum field theory is the energy of the "vacuum" - that is, of the ground state of a field subjected to some nontrivial external condition [13, 10, 9, 18, 39, 41, 8. (The prototype is the electromagnetic field between two parallel flat conductors.) Although the only quantities indisputably open to experiment are the derivatives of the total energy with respect to parameters defining the configuration, the energy itself and even its localization in space are of theoretical interest, not least because energy density (along with associated quantities such as pressure) acts as the source of the gravitational field in general relativity $[\mathbf{2 3}, \mathbf{1 8}]$.

From a mathematical point of view, vacuum energy is a probe of the spectral properties of a self-adjoint differential operator, say $H$; it contains "nonlocal" information not extractible from the much-studied small-time expansion of the heat kernel [36, 29. It reflects the oscillatory fine structure of the eigenvalue distribution and, therefore, is directly related to the spectrum of periodic orbits 32, 2, 16, 14, 20, 15, 17, 45, of the nonrelativistic classical-mechanical (or rayoptical) system associated with our operator $H$ as quantum Hamiltonian (or wave

2000 Mathematics Subject Classification. Primary 81Q10; Secondary 34B45, 81V10.

Key words and phrases. quantum graph, vacuum, spectral density, Robin.

(C)0000 (copyright holder) 
operator). The interplay among spectral theory, dynamics, and vacuum energy is fascinating and rapidly developing, with each subject gaining benefits from the others [10, 3, 43, 40, 25, 35. Inasmuch as quantum graphs provide instructive models of spectral theory and semiclassical dynamics, they should also be communicating with vacuum energy.

Here we show that some elementary techniques recently applied [6] to certain traditional boundary-value problems of the Robin type actually apply also to quantum graphs. Indeed, they may be more valuable there, because of quantum graph theory's supply of nontrivial problems susceptible to essentially one-dimensional methods.

\section{Infinite star graphs}

Here we will consider only the simplest type of quantum graph, one consisting of a single vertex with $N$ infinite edges attached. (Since many of the issues we will study are basically local, there is a sense in which these graphs are the building blocks for all others.) The Hilbert space of the model thus consists of vector-valued functions, $u=\left\{u_{j}(x)\right\} \in L^{2}(0, \infty)^{N}$, where $x$ as the argument of $u_{j}$ is the distance of the point in question from the vertex along edge $j$.

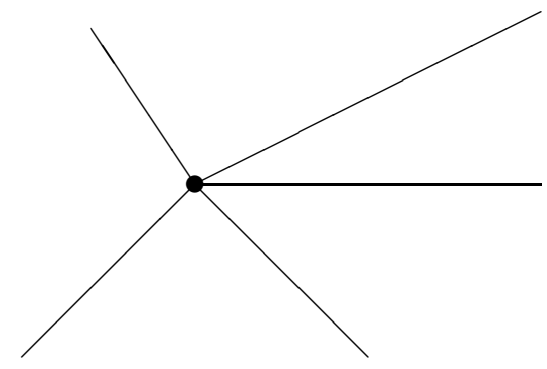

The self-adjoint operator is $H=-\frac{d^{2}}{d x^{2}}$ with certain boundary conditions. We impose the usual continuity conditions,

$$
u_{j}(0)=u(0), \quad \forall j=1, \ldots, N .
$$

The remaining condition will be one of these:

- the Dirichlet condition, $u(0)=0$;

- the Kirchhoff, or generalized Neumann, condition,

$$
\sum_{j=1}^{N} u_{j}^{\prime}(0)=0 ;
$$

- our main concern, the Exner-Šeba or generalized Robin condition

$$
\sum_{j=1}^{N} u_{j}^{\prime}(0)=\alpha u(0), \quad \alpha>0 .
$$

Condition (3) was apparently introduced in [22]. It is often [21, 38 called the $\delta$ condition because it can be regarded as the effect of attaching a Dirac delta potential at the vertex. At a vertex with only one edge it reduces to the so-called Robin (or convective cooling) condition, $u^{\prime}(0)=\alpha u(0)$. In passing we remark that the label "Robin" has almost no historical justification [31, but it is preferable to "mixed" because "mixed boundary conditions" has acquired other meanings [36, 29]. 
Remark. The case $\alpha<0$ can also be handled, but the construction of the Bondurant transform (4) is then so different as to require a separate discussion. With our sign convention, $\alpha \geq 0$ is the more "physical" case, where heat flows from the hotter to the cooler and $H$ has no negative eigenvalues.

\section{The Bondurant transform}

In 6] we showed how to obtain solutions of the simplest problems with Robin boundary conditions from solutions of the corresponding Dirichlet problems. The term "Dirichlet-to-Robin transform" has sometimes been misunderstood as referring to an analogue of the Dirichlet-to-Neumann map, hence the justification for immediately naming the construction after my junior collaborator. Here one is not studying the relation between nonhomogeneous Dirichlet data and nonhomogeneous Neumann data for a fixed solution; instead, one is constructing a new solution to a different problem, with homogeneous Robin (or Exner-Šeba) data replacing Dirichlet data.

The generalization of the key formula of [6 to an infinite star graph with boundary conditions (11) and (3) is

$$
\begin{aligned}
u_{j}(x) & =\left(T^{-1} v\right)_{j}(x) \\
& \equiv \frac{1}{\alpha}\left[\frac{1}{N} \sum_{k=1}^{N} v_{k}(x)-v_{j}(x)\right]-\frac{1}{N^{2}} \int_{x}^{\infty} e^{-\alpha(s-x) / N} \sum_{k=1}^{N} v_{k}(s) d s .
\end{aligned}
$$

THEOREM. If $v(t, x)$ solves a Dirichlet problem for a constant-coefficient partial differential equation [cf. (6) - (9)], then $u(t, x)$ solves the corresponding Exner-Šeba problem (though with different initial data).

SKetch of VERIFICATION. (4) is obtained by solving the ordinary differential equation $v=T u$, where

$$
(T u)_{j}(x) \equiv \sum_{k=1}^{N} u_{k}^{\prime}(x)-\alpha u_{j}(x),
$$

with the condition of decay as $x \rightarrow \infty$. The heuristics of finding the solution are less instructive than the verification that it satisfies all the required conditions. Since $v(0)=0$, one observes that

- $u_{j}(0)$ is independent of $j$ (condition (11));

- $T u(x)=v(x)$, so $\sum_{j=1}^{N} u_{j}^{\prime}(0)=\alpha u(0)($ condition (3) $)$;

- $T$ and $T^{-1}$ commute with the partial differential operator, so $u$ is still a solution.

\section{Integral kernels}

Now consider any of the following initial-value problems.

$$
\begin{array}{ll}
\text { Wave: } & u_{t t}=u_{x x}, \quad u(0, x)=f(x), u_{t}(0, x)=0 \\
\text { Heat: } & u_{t}=u_{x x}, \quad u(0, x)=f(x) \\
\text { Quantum: } & i u_{t}=-u_{x x}=H u, \quad u(0, x)=f(x) \\
\text { Cylinder: } & u_{t t}=H u, \quad u(0, x)=f(x), u(+\infty, 0)=0
\end{array}
$$


(Of course, there are many other problems involving the operator $H$ on the graph that could be considered, but these form a natural and highly useful quartet.) We seek the integral kernel (Green function) that solves such a problem via

$$
u_{j}(t, x)=\sum_{l=1}^{N} \int_{0}^{\infty} G_{S j}^{l}(t, x, y) f_{l}(y) d y .
$$

Corollary. Let $G_{S}(t, x, y)$ be the (matrix) Green function for one of the initial-value problems (6)-(2) on the graph. Let $G(t,|x-y|)$ be the corresponding (scalar) Green function on the real line (also known as the "free" kernel). Then

$$
\begin{aligned}
G_{S j}{ }^{l}(t, x, y) & =\delta_{j}^{l} G(t,|x-y|) \\
& +\left(\frac{2}{N}-\delta_{j}^{l}\right) G(t, x+y)-\frac{2 \alpha}{N^{2}} \int_{x}^{\infty} e^{-\alpha(s-x) / N} G(t, s+y) d s
\end{aligned}
$$

Sketch of DeRivation. The Dirichlet Green function consists of an incident term minus an image term,

$$
\begin{aligned}
G_{D j}^{l}(t, x, y) & =\delta_{j}^{l}[G(t,|x-y|)-G(t, x+y)] \\
& \equiv G_{-}-G_{+} .
\end{aligned}
$$

In operator language, we want $G_{S}=T^{-1} G_{D} T$. (The final $T$ is needed to get the correct initial value, $\delta_{j}^{l} \delta(x-y)$.) In kernel language, therefore, we need

$$
G_{S}(t, x, y)=T_{x}^{-1} T_{y}^{\dagger} G_{D}(t, x, y),
$$

where $\uparrow$ indicates the transpose (real adjoint). But

$$
\begin{aligned}
\left(T^{\dagger} u\right)_{j} & =-\sum_{k} u_{k}^{\prime}-\alpha u_{j} \\
& =-(T u)_{j}-2 \alpha u_{j} .
\end{aligned}
$$

From (12) and $-\partial_{y} G(t,|x-y|)=+\partial_{x} G(t,|x-y|)$ one sees that the incident term passes through the similarity transformation unchanged: $T^{-1} G_{-} T=G_{-}$. From (13) and $-\partial_{y} G(t, x+y)=-\partial_{x} G(t, x+y)$ one has $T_{y} G_{+}=T_{x} G_{+}$and hence

$$
G_{S j}^{l}(t, x, y)=\delta_{j}^{l}[G(t,|x-y|)+G(t, x+y)]+2 \alpha T_{x, j}^{-1}\left[\delta_{j}^{l} G(t, x+y)\right] .
$$

(Note that the first two terms of (14) solve the true Neumann (not Kirchhoff) problem, $u_{j}^{\prime}(0)=0 \forall j$.) Working out the last term of (14) according to (4), one obtains (11).

The factor $\left(\frac{2}{N}-\delta_{j}^{l}\right)$ in (11) will come as no surprise to those who are familiar with the study of quantum graphs by other methods (see [37]).

REMARK. The kernel formula (11) is correct for $\alpha=0$ (the Kirchhoff condition), although the intermediate steps are meaningless (in particular, $T^{-1}$ doesn't exist in that case).

MAIN RESUlts. As corollaries of the corollary, we derive formulas (16)-(17), (22), (25), (27), (35), (36) for particular kernels and associated quantities. 


\section{The wave kernel}

In a one-dimensional system the simplest member of the quartet is the wave problem (6), for which the free Green function is (d'Alembert's solution)

$$
G(t, z)=\frac{1}{2}[\delta(z-t)+\delta(z+t)] .
$$

Applying (11) and omitting terms that vanish for $t>0$ one gets

$$
\begin{aligned}
G_{S j}^{l}(t, x, y)= & \frac{1}{2} \delta_{j}^{l}[\delta(x-y-t)+\delta(x-y+t)] \\
& +\frac{1}{2}\left(\frac{2}{N}-\delta_{j}^{l}\right) \delta(x+y-t)-\frac{\alpha}{N^{2}} e^{-\alpha(t-y-x) / N} \theta(t-y-x),
\end{aligned}
$$

where $\theta$ is the unit step function.

The meaning of (16) becomes clearer when one applies (10) to get

$$
\begin{aligned}
u_{j}(t, x)= & \frac{1}{2}\left[f_{j}(x-t)+f_{j}(x+t)\right]-\frac{1}{2} f_{j}(t-x) \\
& +\frac{1}{N} \sum_{l=1}^{N} f_{l}(t-x)-\frac{\alpha}{N^{2}} \theta(t-x) \int_{0}^{t-x} e^{-\alpha \epsilon / N} \sum_{l=1}^{N} f_{l}(t-x-\epsilon) d \epsilon .
\end{aligned}
$$

Here we see clearly the incident wave, the immediately reflected and transmitted waves from a Kirchhoff vertex, and some $\alpha$-dependent delayed transmission. The Robin case $(N=1)$ is $[\mathbf{6}$

(18) $u(t, x)=\frac{1}{2}[f(x-t)+f(x+t)+f(t-x)]-\alpha \theta(t-x) \int_{0}^{t-x} e^{-\alpha \epsilon} f(t-x-\epsilon) d \epsilon$.

What is the physical meaning of this time delay? In the context of the wave equation, the Robin or Exner-Šba boundary models an ideal spring, or elastic support, to which the vibrating medium is attached. The spring absorbs energy from the medium and leaks it back out. It is a jolly exercise in integration by parts to show that for the solution (18) the total energy

$$
E=\frac{1}{2} \int_{0}^{\infty}\left[\left(\frac{\partial u}{\partial t}\right)^{2}+\left(\frac{\partial u}{\partial x}\right)^{2}\right] d x+\frac{\alpha}{2} u(t, 0)^{2}
$$

is indeed conserved; the field and boundary terms individually are not (unless $\alpha=0)$.

For later use we note that one integration by parts in (19) and use of $u^{\prime}(t, 0)=$ $\alpha u(t, 0)$ lead to an alternative formula for the total energy,

$$
E=\frac{1}{2} \int_{0}^{\infty}\left[\left(\frac{\partial u}{\partial t}\right)^{2}-u\left(\frac{\partial^{2} u}{\partial x^{2}}\right)\right] d x
$$

in which the boundary term has been formally absorbed into the field term.

\section{The spectral projection kernel}

Let $P(\lambda)$ be the orthogonal projection operator onto the interval $[0, \lambda]$ of the spectral resolution of $H$. Because $H$ on an infinite star graph has purely absolutely continuous (and nonnegative) spectrum, the integral kernel of $P(\lambda)$ may be written

$$
P(\lambda, x, y)=\int_{0}^{\sqrt{\lambda}} \sigma(\omega, x, y) d \omega
$$


where $\sigma$ is a well-defined matrix-valued function (not just a distribution in $\omega$ ). Alternatively, $\sigma$ can be defined as the inverse Fourier cosine transform of the wave kernel. (It is also the inverse Laplace transform of the heat kernel, and so on for all the standard "spectral functions" [36.)

That is, we write

$$
G_{S j}^{l}(t, x, y)=\int_{0}^{\infty} \cos (\omega t) \sigma_{S j}^{l}(\omega, x, y) d \omega
$$

and calculate

$$
\begin{aligned}
\sigma_{S j}{ }^{l}(\omega, x, y)= & \frac{2}{\pi} \int_{0}^{\infty} \cos (\omega t) G_{S j}{ }^{l}(t, x, y) d t \\
= & \frac{2}{\pi} \delta_{j}^{l} \sin (\omega x) \sin (\omega y) \\
& +\frac{2 / \pi}{\alpha^{2}+N^{2} \omega^{2}}\left\{N \omega^{2} \cos [\omega(x+y)]+\alpha \omega \sin [\omega(x+y)]\right\} .
\end{aligned}
$$

In a sense, (22) is the ultimate formula concerning the operator $H$, since all operator functions of $H$ can be calculated from it in principle and it contains all facts about the spectral resolution in a rather explicit form.

Kottos and Smilansky 37. Sec. 3B] found the spectral resolution by treating the infinite star graph as a scattering problem. For each $\omega$ they provide the basis of incoming scattering eigenfunctions

$$
\begin{aligned}
\psi_{j}^{l}(x) & \equiv \delta_{j}^{l} e^{-i \omega x}+\left[-\delta_{j}^{l}+\frac{1}{N}\left(1+e^{-2 i \tan ^{-1} \frac{\alpha}{N \omega}}\right)\right] e^{i \omega x} \\
& =-2 i \delta_{j}^{l} \sin (\omega x)+2 \omega \frac{N \omega-i \alpha}{\alpha^{2}+N^{2} \omega^{2}} e^{i \omega x} .
\end{aligned}
$$

These basis elements are orthonormal (up to the conventional factor $\sqrt{2 \pi}$ ) and therefore

$$
\frac{1}{2 \pi} \sum_{l=1}^{N} \psi_{j}{ }^{l}(x) \psi_{j^{\prime}}{ }^{l}(y)^{*}=\sigma_{S j}{ }^{j^{\prime}}(\omega, x, y) .
$$

A calculation verifies that (22) and (24) agree.

REMARK: Direct construction of eigenfunctions by applying the Bondurant transform to orthonormal eigenfunctions of the Dirichlet problem, while possible, is not recommended. In the present problem the immediate results are not orthogonal, much less normalized. If $\alpha=0$ they are not even linearly independent (because $T$ is not invertible), and one basis element needs to be found by a separate argument.

\section{The local spectral density}

Special interest attaches to the diagonal values of $\sigma$,

$$
\begin{aligned}
\sigma_{S j}{ }^{j}(\omega, x, x)=\frac{1}{\pi}+\frac{1}{\pi}\left(\frac{2}{N}-1\right) & \cos (2 \omega x) \\
& +\frac{2 \alpha / \pi}{\alpha^{2}+N^{2} \omega^{2}}\left[\omega \sin (2 \omega x)-\frac{\alpha}{N} \cos (2 \omega x)\right]
\end{aligned}
$$

Clearly here the $\frac{1}{\pi}$ is the universal Weyl term for a one-dimensional system, the next term is the spectral effect of a Kirchhoff vertex, and the last term is the Exner-Seba 
correction. In the limit $\alpha \rightarrow+\infty$ there is some cancellation between the second and third terms, resulting in

$$
\frac{1}{\pi}-\frac{1}{\pi} \cos (2 \omega x)=\sigma_{D j}{ }^{j}(\omega, x, x) .
$$

as expected for a Dirichlet vertex.

Because the spectrum is continuous, one can't integrate (25) to get a density of states. However, subtracting off the Weyl term and paying due attention to distributional limits, one can obtain a meaningful global spectral density:

$$
\begin{aligned}
\Delta \rho(\omega) & \equiv \int_{0}^{\infty} \sum_{j=1}^{N}\left[\sigma_{S j}{ }^{j}(\omega, x, x)-\frac{1}{\pi}\right] d x \\
& =\left(\frac{1}{2}-\frac{N}{4}\right) \delta(\omega)+\left[\frac{N \alpha / \pi}{\alpha^{2}+N^{2} \omega^{2}}-\frac{1}{2} \delta(\omega)\right] .
\end{aligned}
$$

This expression approximates the incremental effect that such a vertex would have in a problem with discrete spectrum. The first term in (27) is the Kirchhoff term; it vanishes when $N=2$, because a Kirchhoff vertex with exactly two edges is vacuous. The other term is the Exner-Šeba correction; it vanishes when $\alpha=0$ because its first term distributionally approaches $\frac{1}{2} \delta(\omega)$ in that limit. Alternatively, (27) can be simplified to

$$
\Delta \rho(\omega)=-\frac{N}{4} \delta(\omega)+\frac{N \alpha / \pi}{\alpha^{2}+N^{2} \omega^{2}} ;
$$

here the first term is the correct formula for a Dirichlet vertex and the remaining term is $O\left(\alpha^{-1}\right)$ as $\alpha \rightarrow \infty$.

REMARK: The meaning of a Dirac delta distribution in formulas such as (27) and (28) is that the spectral counting function $N(\omega)$ has a nonzero limit as $\omega$ approaches 0 from above $(N(\omega)$ being understood to be 0 for negative $\omega)$. For example, (28) is simply the derivative of the formula

$$
\Delta N(\omega)=\left[-\frac{N}{4}+\frac{1}{\pi} \tan ^{-1} \frac{N \omega}{\alpha}\right] \theta(\omega)
$$

for the incremental effect of the vertex on the total number of eigenvalues in the interval $0 \leq \lambda \leq \omega^{2}$.

The Bondurant method cannot be applied directly to a finite interval, because no transformation $T$ will work for both boundary conditions simultaneously. However, the appropriate operators $T^{-1}$ for the two boundaries can be applied alternately to construct a solution as an infinite series (a sum over closed classical paths, generalizing the classic method of images). In $\mathbf{6}$ the wave kernel and hence the local and global spectral densities were obtained in that way for a finite interval with one Robin and one Dirichlet endpoint. Numerical evaluation reveals the correct eigenvalues for the problem emerging as spikes in the global density (the counterpart of (27)). See [44 for a related study in two dimensions. It should be straightforward to extend this analysis (and also the study of vacuum energy, etc.) to an arbitrary finite star graph, and in principle to more complicated quantum graphs. It is noteworthy that in these systems no semiclassical (or stationary-phase) approximation is needed to obtain the representation of the spectrum in terms of classical paths; the only approximation involved is the truncation of the sum at some maximum path length if and when one resorts to numerics. 


\section{Heat and quantum kernels}

The same machine (11) can be used to treat the problems (7) and (8), for which the free kernels are

$$
G(t, z)=(4 \pi t)^{-1 / 2} e^{-z^{2} / 4 t}, \quad G(t, z)=(4 \pi i t)^{-1 / 2} e^{-z^{2} / 4 i t},
$$

respectively. (The results are qualitatively similar to (35) below, with the complementary error function appearing instead of the exponential integral function.) Studying the heat kernel is the traditional route to equations like (29) for partial differential operators. In one-dimensional systems such as quantum graphs, however, the wave kernel built from (15) appears to be easier to calculate.

Using the heat kernel, the Robin case of (28) was obtained in 6 Secs. 3.3 and 5.5]. That analysis extends to flat boundaries with constant $\alpha$ in any dimension. The results fit naturally with those of 1 in dimension 3 and 44 in dimension 2, where the leading orders in boundary curvature are also included. All these formulas are exact in $\alpha$; of course, when the heat-kernel formulas are expanded in power series in $\alpha$ they match and extend the relevant terms tabulated in such references as [36, 29. (See also [1, 7.) Apart from a unifying point of view, it is not claimed that these results are particularly new; in fact, the Robin heat kernels in dimensions 1 and 3 were found in 1891 by a closely related method [1, 12.

\section{Vacuum energy density}

First, a paragraph which pure mathematicians are free to ignore: From a physical point of view, vacuum energy involves a relativistic (usually massless and bosonic) field. (It is of no relevance, therefore, to quantum graphs if they are regarded solely as models of nonrelativistic electrons in networks of wires.) Formally, the total energy corresponding to the wave operator $H$ is

$$
E=\frac{1}{2} \sum_{n=1}^{\infty} \omega_{n}=\frac{1}{2} \int_{0}^{\infty} \omega \rho(\omega) d \omega
$$

for an operator with purely point spectrum. (To avoid irrelevant complications, let us also always assume that $H$ has no negative spectrum.) Equally formally, the local energy density is

$$
T_{00}(x)=\frac{1}{2} \int_{0}^{\infty} \omega \sigma(\omega, x, x) d \omega
$$

(without the requirement of point spectrum). The origin of these expressions is the "second-quantized" theory of a field satisfying the wave equation (6), in which each normal mode of the field (with frequency $\omega$ ) becomes a quantum harmonic oscillator (with ground state energy $\frac{1}{2} \omega$ ). Then (31) results from the integrand of (20), and (30) comes from integrating (31) over all space or just from adding up the energies of all the modes. Both integrals, (30) and (31), are divergent at the upper limit and are to be defined by a renormalization procedure.

Our claim is that vacuum energy should be of mathematical interest even in models whose physical relevance is questionable. Therefore, we provide here a precise mathematical definition, which incorporates a particular renormalization prescription (whose physical rationale need not concern us): Consider the cylinder kernel (the Green function of (9)) on diagonal $(y=x, l=j)$, find its asymptotic expansion as $t \rightarrow 0$, and extract the coefficient of the term proportional to $t$, 
times $-\frac{1}{2}$; this is the vacuum energy density, $T_{00}(x)$. When appropriate, integrate over $x$ (and sum over $j$ in our graph problem), before taking t to 0 , to define a total energy, $E$.

The intuition behind this definition is the following. Let $G(t, x, y)$ be the cylinder kernel of the problem under study. (For an infinite star graph it is the matrix $\left.G_{S}.\right)$ Then

$$
G(t, x, x)=\int_{0}^{\infty} e^{-\omega t} \sigma(\omega, x, x) d \omega
$$

and (when "appropriate") its trace is

$$
\operatorname{Tr} G(t) \equiv \int_{0}^{\infty} \sum_{j=1}^{N} G_{j}^{j}(t, x, x) d x=\sum_{n=1}^{\infty} e^{-\omega_{n} t} .
$$

Now take $-\frac{1}{2} \frac{\partial}{\partial t}$ of (32) and (33) and let $t$ approach 0, formally obtaining (31) and (30), respectively. Systematically throwing away the terms of negative order in the Laurent expansions (and a possible logarithmic term), one arrives at our definition.

To find the vacuum energy of an infinite star graph, one can apply the Bondurant machine one more time, to the free cylinder kernel

$$
G(t, z)=\frac{t / \pi}{t^{2}+z^{2}}
$$

obtaining

$$
\begin{aligned}
G_{S j}{ }^{l}=\delta_{j}^{l} \frac{t / \pi}{t^{2}+(x-y)^{2}} & +\left(\frac{2}{N}-\delta_{j}^{l}\right) \frac{t / \pi}{t^{2}+(x+y)^{2}} \\
& +\frac{2 \alpha}{\pi N^{2}} e^{\alpha(x+y) / N} \operatorname{Im}\left[e^{-i \alpha t / N} \operatorname{Ei}\left(\frac{i \alpha t}{N}-\frac{\alpha}{N}(x+y)\right)\right] .
\end{aligned}
$$

It follows that

$$
T_{00}(x)=\left(1-\frac{2}{N}\right) \frac{1}{8 \pi x^{2}}+\frac{\alpha}{2 \pi N^{2} x}+\frac{\alpha^{2}}{\pi N^{3}} e^{2 \alpha x / N} \operatorname{Ei}\left(-\frac{2 \alpha x}{N}\right) .
$$

The most important parameter in this problem is the dimensionless product $\alpha x$. Therefore, at short distance the Kirchhoff term dominates:

$$
T_{00}(x) \sim\left(1-\frac{2}{N}\right) \frac{1}{8 \pi x^{2}}+\frac{\alpha}{2 \pi N^{2} x}+\frac{\alpha^{2}}{\pi N^{3}} \ln |\alpha x|+O\left(\alpha^{2} x^{2}\right),
$$

whereas at large distance the energy density is almost pure Dirichlet:

$$
T_{00}(x) \sim \frac{1}{8 \pi x^{2}}+O\left(\alpha^{-1} x^{-3}\right) .
$$

The nonintegrable $O\left(x^{-2}\right)$ singularity in (37) would interfere with calculating a total energy by integration over $x$, even if the edges were finite. The renormalization procedure implicit in our definition does not commute with the spatial integration, however, and it leads to a finite total energy [5, 24, 25. As agreed, we will not delve here into the physical issues thereby raised (which are still somewhat controversial).

In a sense the calculation based on the cylinder kernel was unnecessary, given the spectral formulas (22), (25), and (27). Indeed, (36) can be obtained from (32) and (25) and a Laurent expansion, or even immediately from (31) using the subtracted spectral density appearing in the integrand of (27). (The subtraction of the Weyl term corresponds to the subtraction of the leading Laurent term. In other 
problems, such as higher dimensions, additional subtractions would be necessary.) Similar methods were used in 42 to find the effect of a flat Robin boundary in any dimension. In general, however, it is easier to find the small- $t$ expansion of a cylinder kernel than to find the detailed spectral resolution; as for heat kernels, one expects useful calculations to run in the opposite direction. Romeo and Saharian 42 also gave complicated integral formulas for the vacuum energy and energy density of a finite interval with two Robin boundaries. Our methods will instead give these quantities as infinite sums over classical paths [27.

\section{A broader perspective}

Now let $H$ be a generic differential operator (self-adjoint, elliptic, positive, second-order, with scalar principal symbol) in dimension $d$. Let $T$ be the cylinder kernel and $K$ be the heat kernel. Then

$$
\begin{array}{ll}
\operatorname{Tr} T=\int_{0}^{\infty} e^{-t \omega} d N=\int_{0}^{\infty} e^{-t \omega} \rho(\omega) d \omega, & T(t, x, x)=\int_{0}^{\infty} e^{-t \omega} \sigma(\omega, x, x) d \omega, \\
\operatorname{Tr} K=\int_{0}^{\infty} e^{-t \lambda} d N=\int_{0}^{\infty} e^{-t \omega^{2}} \rho(\omega) d \omega, & K(t, x, x)=\int_{0}^{\infty} e^{-t \omega^{2}} \sigma(\omega, x, x) d \omega,
\end{array}
$$

where $N$ now is the number of eigenvalues less than or equal to $\lambda=\omega^{2}$.

It is known that as $t \rightarrow 0$ the global quantities have expansions of the forms

$$
\begin{gathered}
\operatorname{Tr} T \sim \sum_{s=0}^{\infty} e_{s} t^{-d+s}+\sum_{\substack{s=d+1 \\
s=d \text { odd }}}^{\infty} f_{s} t^{-d+s} \ln t \\
\operatorname{Tr} K \sim \sum_{s=0}^{\infty} b_{s} t^{(-d+s) / 2} .
\end{gathered}
$$

The local quantities, $T(t, x, x)$ and $K(t, x, x)$, have (nonuniform in $x$ ) expansions of precisely the same respective forms; we do not introduce a separate notation for their coefficients. If $d-s$ is even or positive,

$$
e_{s}=\pi^{-1 / 2} 2^{d-s} \Gamma((d-s+1) / 2) b_{s} .
$$

If $d-s$ is odd and negative, then

$$
f_{s}=\frac{(-1)^{(s-d+1) / 2} 2^{d-s+1}}{\sqrt{\pi} \Gamma((s-d+1) / 2)} b_{s},
$$

but, most strikingly, in that case

$$
e_{s} \text { is undetermined by the } b_{r} \text {. }
$$

I have expounded in detail elsewhere 26, 24, 25 how the connections (41)(43) come about: The $b_{s}$ are proportional to coefficients in the high-frequency asymptotics of the Riesz means [33, 34] of $N$ (or of $\int \sigma$ ) with respect to $\lambda$, while the $e_{s}$ and $f_{s}$ are proportional to coefficients in the asymptotics of the Riesz means with respect to $\omega$. Alternatively, they are related through the poles of the zeta functions of $H$ and $\sqrt{H}[\mathbf{2 8}, \mathbf{3 0}, \mathbf{4}$.

The new half of the cylinder-kernel coefficients (those in 433) - of which the first, $e_{d+1}$, is proportional to the vacuum energy - are a new set of moments of the spectral distribution. What are they good for, mathematically? Unlike the old 
ones, they are nonlocal in their dependence on the geometry of the domain and the coefficient functions of $H$. They probe, in a comparatively crude, averaged way, the oscillatory spectral structures that are correlated more precisely with periodic and closed classical paths. Thus they embody, at least partially, the global dynamical structure of the system; they are a half-way house between the heat-kernel coefficients and a full semiclassical closed-orbit analysis.

\section{References}

[1] R. Balian and C. Bloch, Distribution of eigenfrequencies for the wave equation in a finite domain. I, Ann. Phys. 60 (1970) 401-447.

[2] R. Balian and C. Bloch, Distribution of eigenfrequencies for the wave equation in a finite domain. III, Ann. Phys. 69 (1972) 76-160.

[3] R. Balian and B. Duplantier, Electromagnetic waves near perfect conductors. I, II, Ann. Phys. 104 (1977) 300-335; ibid. 112 (1978) 165-208.

[4] C. Bär and S. Moroianu, Heat kernel asymptotics for roots of generalized Laplacians, Internat. J. Math. 14 (2003) 397-412.

[5] F. Bernasconi, G. M. Graf, and D. Hasler, The heat kernel expansion for the electromagnetic field in a cavity, Ann. Henri Poincaré 4 (2003) 1001-1013.

[6] J. D. Bondurant and S. A. Fulling, The Dirichlet-to-Robin transform, J. Phys. A 38 (2005) $1505-1532$.

[7] M. Bordag, H. Falomir, E. M. Santangelo, and D. V. Vassilevich, Boundary dynamics and multiple reflection expansion for Robin boundary conditions, Phys. Rev. D 65 (2002) 064032.

[8] M. Bordag, U. Mohideen, and V. M. Mostepanenko, New developments in the Casimir effect, Phys. Reps. 353 (2001) 1-205.

[9] T. H. Boyer, Quantum zero-point energy and long-range forces, Ann. Phys. 56 (1970) 474503.

[10] L. S. Brown and G. J. Maclay, Vacuum stress between conducting plates: An image solution, Phys. Rev. 184 (1969) 1272-1279.

[11] G. H. Bryan, Note on a problem in the linear conduction of heat, Proc. Camb. Phil. Soc. 7 (1891) 246-248.

[12] G. H. Bryan, An application of the method of images to the conduction of heat, Proc. London Math. Soc. 22 (1891) 424-430.

[13] H. B. G. Casimir, On the attraction between two perfectly conducting plates, Konink. Nederl. Akad. Wet., Proc. Sec. Sci. 51 (1948) 793-795.

[14] J. Chazarain, Formule de Poisson pour les variétés riemanniennes, Invent. Math. 24 (1974) 65-82.

[15] D. Cohen, H. Primack, and U. Smilansky, Quantal-classical duality and the semiclassical trace formula, Ann. Phys. 264 (1998) 108-170.

[16] Y. Colin de Verdière, Spectre du Laplacien et longueurs des géodésiques périodiques. II, Compos. Math. 27 (1973) 159-184.

[17] M. Combescure, J. Ralston, and D. Robert, A proof of the Gutzwiller semiclassical trace formula using coherent states decomposition, Commun. Math. Phys. 202 (1999) 463-480.

[18] D. Deutsch and P. Candelas, Boundary effects in quantum field theory, Phys. Rev. D 20 (1979) 3063-3080.

[19] J. S. Dowker, Robin conditions on the Euclidean ball, Class. Quantum Grav. 13 (1996) 585610.

[20] J. J. Duistermaat and V. W. Guillemin, The spectrum of positive elliptic operators and periodic bicharacteristics, Invent. Math. 29 (1975) 39-79.

[21] P. Exner, Lattice Kronig-Penney models, Phys. Rev. Lett. 74 (1995) 3503-3506.

[22] P. Exner and P. Šeba, Free quantum motion on a branching graph, Reps. Math. Phys. 28 (1989) $7-26$

[23] L. H. Ford, Quantum vacuum energy in general relativity, Phys. Rev. D 11 (1975) 3370-3377.

[24] S. A. Fulling, Systematics of the relationship between vacuum energy calculations and heatkernel coefficients, J. Phys. A 36 (2003) 6857-6873. 
[25] S. A. Fulling, Global and local vacuum energy and closed orbit theory, in Quantum Field Theory Under the Influence of External Conditions, ed. by K. A. Milton, Rinton, Princeton, 2004, pp. 166-174.

[26] S. A. Fulling and R. A. Gustafson, Some properties of Riesz means and spectral expansions, Electronic J. Diff. Eqs. 1999, No. 6 (1999) 1-39.

[27] S. A. Fulling and Z. Liu, work in progress.

[28] P. B. Gilkey, The spectral geometry of the higher order Laplacian, Duke Math. J. 47 (1980) $511-528$.

[29] P. B. Gilkey, Asymptotic Formulae in Spectral Geometry, Chapman \& Hall/CRC, Boca Raton, 2004.

[30] P. B. Gilkey and G. Grubb, Logarithmic terms in asymptotic expansions of heat operator traces, Commun. Partial Diff. Eqs. 23 (1998) 777-792.

[31] K. Gustafson and T. Abe, The third boundary condition - Was it Robin's?, Math. Intelligencer 20, No. 1 (1998) 63-71.

[32] M. C. Gutzwiller, Periodic orbits and classical quantization conditions, J. Math. Phys. 12 (1971) 343-358.

[33] G. H. Hardy, The second theorem of consistency for summable series, Proc. London Math. Soc. 15 (1916) 72-88.

[34] L. Hörmander, The spectral function of an elliptic operator, Acta Math. 121 (1968) 193-218.

[35] R. L. Jaffe and A. Scardicchio, Casimir effects: An optical approach. I, Nucl. Phys. B 704 (2005) 552-582.

[36] K. Kirsten, Spectral Functions in Mathematics and Physics, Chapman \& Hall/CRC, Boca Raton, 2002.

[37] T. Kottos and U. Smilansky, Periodic orbit theory and spectral statistics for quantum graphs, Ann. Phys. 274 (1999) 76-124.

[38] P. Kuchment, Quantum graphs. I, Waves Random Media 14 (2004) S107-S128.

[39] S. K. Lamoreaux, Resource letter CF-1: Casimir force, Amer. J. Phys. 67 (1999) 850-861.

[40] F. D. Mazzitelli, M. J. Sánchez, N. N. Scoccola, and J. von Stecher, Casimir interaction between two concentric cylinders: Exact versus semiclassical results, Phys. Rev. A 67 (2003) 013807.

[41] K. A. Milton, The Casimir Effect: Physical Manifestations of Zero-Point Energy, World Scientific, Singapore, 2001.

[42] A. Romeo and A. A. Saharian, Casimir effect for scalar fields under Robin boundary conditions on plates, J. Phys. A 35 (2002) 1297-1320.

[43] M. Schaden and L. Spruch, Infinity-free semiclassical evaluation of Casimir effects, Phys. Rev. A 58 (1998) 935-953.

[44] M. Sieber, H. Primack, U. Smilansky, I. Ussishkin, and H. Schanz, Semiclassical quantization of billiards with mixed boundary conditions, J. Phys. A 28 (1995) 5041-5078.

[45] A. Uribe, Trace formulae, in First Summer School in Analysis and Mathematical Physics: Quantization, the Segal-Bargmann Transform and Semiclassical Analysis (Contemp. Math. 260), ed. by S. Pérez-Esteva and C. Villegas-Blas, American Mathematical Society, Providence, 2000, pp. 61-90.

Departments of Mathematics and Physics, Texas A\&M University, College Station, TX, 77843-3368

E-mail address: fulling@math.tamu.edu 